\title{
"Pulling telescoped phantoms out of the stump": Manipulating the perceived position of phantom limbs using a full-body illusion
}

\author{
Laura SchmalzI $^{1}{ }^{*}$, Erik Thomke $^{1}$, Christina Ragnö $^{2}$, Maria Nilseryd $^{2}$, Anita Stockselius $^{2}$ and \\ H. Henrik Ehrsson ${ }^{1}$
}

Brain, Body and Self Laboratory, Department of Neuroscience, Karolinska Institute, Stockholm, Sweden

${ }^{2}$ Arm Prosthesis Unit, Red Cross Hospital, Stockholm, Sweden

Edited by:

Hauke R. Heekeren, Freie Universität Berlin, Germany

Reviewed by:

Burkhard Pleger, Max Planck Institute, Germany

Herta Flor, Central Institute of Mental Health, Germany

\section{*Correspondence:}

Laura Schmalzl, Brain, Body and Self

Laboratory, Department of

Neuroscience, Karolinska Institute,

Retzius Väg 8, 17177 Stockholm,

Sweden.

e-mail: laura.schmalz|@ki.se
Most amputees experience phantom limbs, or the sensation that their amputated limb is still attached to the body. Phantom limbs can be perceived in the location previously occupied by the intact limb, or they can gradually retract inside the stump, a phenomenon referred to as "telescoping". Telescoping is relevant from a clinical point of view, as it tends to be related to increased levels of phantom pain. In the current study we demonstrate how a full-body illusion can be used to temporarily revoke telescoping sensations in upper limb amputees. During this illusion participants view the body of a mannequin from a first person perspective while being subjected to synchronized visuo-tactile stimulation through stroking, which makes them experience the mannequin's body as their own. In Experiment 1 we used an intact mannequin, and showed that amputees can experience ownership of an intact body as well as referral of touch from both hands of the mannequin. In Experiment 2 and 3 we used an amputated mannequin, and demonstrated that depending on the spatial location of the strokes applied to the mannequin, participants experienced their phantom hand to either remain telescoped, or to actually be located below the stump. The effects were supported by subjective data from questionnaires, as well as verbal reports of the perceived location of the phantom hand in a visual judgment task. These findings are of particular interest, as they show that the temporary revoking of telescoping sensations does not necessarily have to involve the visualization of an intact hand or illusory movement of the phantom (as in the rubber hand illusion or mirror visual feedback therapy), but that it can also be obtained through mere referral of touch from the stump to the spatial location corresponding to that previously occupied by the intact hand. Moreover, our study also provides preliminary evidence for the fact that these manipulations can have an effect on phantom pain sensations.

Keywords: phantom limbs, telescoping, phantom pain, plasticity, full-body illusion

\section{INTRODUCTION}

Up to $98 \%$ of amputees experience phantom limb sensations (Ramachandran and Hirstein, 1998). That is, subsequent to amputations most patients continue to have the sensation that their amputated limb is still attached to the body.

Initially, phantom limbs are mostly perceived to be continuous with the stump, and hence in the same spatial location the intact limb used to be in. Over time however, phantom limbs often retract inside the stump, a phenomenon referred to as "telescoping" (Giummarra et al., 2007). In that case, the proximal portion of the phantom is perceived to be missing or have shrunken, so that the more distal portion of the phantom is perceived as information floating near or actually being inside the stump (Flor et al., 2006). It has been proposed that telescoping occurs because the proximal portions of limbs are less extensively represented in the cortex relative to the distal portions. This proposal is supported by the fact that lower limbs tend to telescope more rapidly than upper limbs (which are more diffusely represented throughout the cortex given their integral role in fine motor tasks; Ramachandran and Hirstein, 1998). The onset and temporal dynamics of telescoping vary greatly across amputees. Typically, telescoping begins within the first weeks following the amputation and progresses gradually over a number of years. However, some patients also describe a sudden occurrence of the phenomenon, with their phantoms literally telescoping overnight (Carlen et al., 1978; Giummarra et al., 2007).

From a clinical perspective, telescoping is of interest as it tends to be related to increased levels of phantom pain (Flor et al., 2006). As telescoping, phantom pain typically emerges immediately, but may also emerge only after months or even years post amputation (Ramachandran and Hirstein, 1998). It can manifest in different ways, including burning, cramping, or tingling sensations (Kooijman et al., 2000). The exact nature of the relationship between telescoping and phantom pain is not yet well understood. It has been put forward that a main factor underlying their co-occurrence could be a mismatch between the sensory 
feedback from the phantom and the cortical regions representing the limb (Flor et al., 2006). That is, while the perception of an extended phantom continues to provide sensory feedback to the brain areas that previously represented the amputated limb, telescoped phantoms activate brain areas remote from the original limb representation. It is this latter cortical re-organization that is hypothesized to underlie the development of increased levels of phantom pain.

In a recent study conducted in our lab (Schmalzl and Ehrsson, 2011), we have demonstrated how the sensation of a telescoped limb can also be induced in healthy individuals in the context of a virtual reality setup involving head-mounted displays (HMDs) connected to video cameras. Specifically, we used a so-called "full-body illusion" (Petkova and Ehrsson, 2008), an experimental manipulation that causes participants to experience an artificial body as their own. For the induction of this illusion, participants see the body of a mannequin from a first person perspective while being subjected to synchronized visuo-tactile stimulation through stroking (as depicted in Figure 2). Crucially, in our experiments, the mannequin was missing one hand so as to resemble an amputee. By manipulating the positioning of the strokes applied to the mannequin's stump with respect to the participants' hand we were then able to clearly manipulate the perceived location of the hand: (a) simultaneous stroking of the participants' hand and the empty space below the mannequin's stump evoked the sensation of the hand being located below the stump; (b) simultaneous stroking of the participants' hand and the end of the mannequin's stump evoked the sensation of the hand being located "inside" the stump, and hence telescoped.

Given these intriguing findings, we embarked on further investigations that are described in the current paper. Namely, we set out to investigate whether the experimental setup used in our study with healthy participants could also be used to manipulate the perceived position of phantom limbs in amputees, with a possible consequence of altering phantom pain sensations. Our rationale for postulating the feasibility of these investigations was motivated by a number of findings from previous literature. Firstly, with regard to the nature of the experimental procedures, it has been shown that upper limb amputees can be induced to experience an artificial hand as their own through synchronous visuo-tactile stimulation. Specifically, Ehrsson et al. (2008) demonstrated that following simultaneous stroking of the amputees' stump and an artificial rubber hand, some patients reported strong referred sensations of touch from, as well as ownership of, the artificial hand. Secondly, with regard to the manipulation of phantom pain sensations, it has been shown that visual illusions can be effective. Specifically, it has been demonstrated that mirror visual feedback (MVF) can be used to alleviate phantom pain (Ramachandran et al., 1995, 2009; Chan et al., 2007). In short, during the MVF amputees view the reflection of their intact hand in a mirror so that it is optically projected onto the location of their missing hand. Consequently, movements performed with the intact hand create simultaneous illusory movements of the now "visible" phantom hand, and such illusory sensations have been found to significantly reduce phantom pain in some patients.

So, based on the findings that amputees can experience sensations of touch that they observe on an artificial hand, and that visual illusions can reduce phantom pain, we hypothesized that the full-body illusion could also be used to induce ownership sensations of an artificial body in amputees, with the possible consequence of altering phantom sensations. With respect to these previous investigations however, the use of the full-body would be novel in a number of ways. Firstly, if reproducible with amputees, the procedures described in Schmalzl and Ehrsson (2011) would allow one to directly manipulate the degree of telescoping of phantom limbs, and have amputees experience touch on their phantom while it is perceived to be located either inside or outside the stump according to the experimental manipulations. While the actualization of this manipulation would be a novelty in its own right, it would also enable one to disentangle potential effects on phantom pain sensations driven by perception of touch on the phantom as such, from those driven by the perceived displacement of the phantom. Secondly, both the rubber hand illusion and the MVF involve visualization of the phantom. That is, in both techniques amputees are induced to experience their phantom in its original location with the aid of a visible hand, either an artificial hand or the mirror image of the individuals' intact hand. In contrast, the full-body illusion with the use of an amputated mannequin would enable the investigation of whether the same effect can be obtained without visualization of an intact hand, but merely through evoking referred touch from a location in space corresponding to the original position of the intact hand. Thirdly, the effects of the MVF on phantom pain have always been explained by emphasizing the crucial importance of the perceived movement of the phantom. We envisaged that our setup would lend itself very well to the examination of the effects of perceived touch on the phantom as opposed to movement of the phantom. And while referred touch as such has already been demonstrated by Ehrsson et al. (2008), their study did not include any documentation of whether this procedure had any effect on phantom pain.

Hence, with this context in mind, in the current study we set out to address the following questions: 1 . Can amputees experience ownership of a whole intact body in the context of the full-body illusion, including referral of touch from, and ownership of, the artificial hand on the amputated side? 2. Can the full-body illusion with an amputated mannequin be used to manipulate the perceived position of phantom limbs, and more specifically to temporarily revoke telescoping sensations without involving any visualization of the phantom? 3. Do any of these experimental procedures have an effect on phantom pain sensations?

\section{MATERIALS AND METHODS PARTICIPANTS}

Ten upper limb amputees were initially recruited through the Arm Prosthesis Unit of Red Cross Hospital, Stockholm, Sweden. All patients had been in contact with the clinic prior to our study, and specifically selected according to the location of their amputation, i.e., below the elbow. Two of them had to be excluded from the study - one because he did not experience any phantom sensations (see exclusion criteria below), and the other because he did not feel well on the day of testing and was therefore not able to complete the experiments. Hence, our final group of participants consisted of eight amputees (Age: 23-65, Mean = 50; Gender: 7 male, 1 female). All of them had lost their limb due to a traumatic 
accident, and none had any significant medical history apart from the amputation. The main inclusion criteria were: (a) location of amputation below the elbow; (b) presence of phantom sensations for at least part of the amputated hand; (c) presence of telescoping. There were no exclusion criteria in regard to age or time since amputation, hence these factors varied significantly across participants (details of all participants are provided in Table 1). The study was approved by the Regional Ethics Review Board of Stockholm, and informed written consent was obtained from all participants.

\section{PRE-EXPERIMENTAL EVALUATIONS}

Prior to the actual experiments all participants underwent a detailed interview, a "stump mapping" procedure, and an evaluation of their phantom pain.

\section{Interview}

A detailed interview was conducted with all participants in order to document their medical history, the details of the accidents that led to their amputation as well as their currently experienced phantom sensations (for a summary see Table $\mathbf{1}$ ).

\section{Stump mapping}

For each participant we also performed a so-called "stump mapping” (Figure 1). This procedure consists of applying systematic touches to the distal portion of the stump in order to determine the exact spots that, when touched, give rise to referred sensations in specific parts of the phantom hand and phantom digits. To facilitate the mapping participants were shown a drawn hand template, which was divided into six colored sections corresponding to each of the individual fingers as well as the central part (palm/back) of the hand. Then, as their stump was being touched, participants were asked to identify any spot on their stump that corresponded to any of the colored sections of the hand template. In addition, they were instructed to point out as best as they could at which exact part of the phantom hand or finger(s) they felt the referred sensations (e.g., knuckle vs. fingertip). These spots where then marked on the stump using markers of the respective colors, and later used as reference points for tactile stimulations during the experiments.

\section{Pain rating scale}

All participants were asked if they experienced any phantom pain sensations, both at the time of the testing session or in general. Participants who reported to experience pain at the time of testing were then asked to rate its intensity on a visual analog scale from 0 to 10 , and describe its characteristics (e.g., cramping, burning, tingling, etc.). The same pain rating scale was then administered after Experiments 1 and 2.

\section{Table 1 | Participants.}

\begin{tabular}{|c|c|c|c|c|c|c|c|c|c|c|}
\hline Participant & Gender & Age & $\begin{array}{l}\text { Time since } \\
\text { amputation } \\
\text { (years) }\end{array}$ & $\begin{array}{l}\text { Side of } \\
\text { amputation }\end{array}$ & $\begin{array}{l}\text { Dominant } \\
\text { hand }\end{array}$ & $\begin{array}{l}\text { Length of } \\
\text { stump from } \\
\text { elbow (cm) }\end{array}$ & $\begin{array}{l}\text { Phantom } \\
\text { hand }\end{array}$ & Telescoping & $\begin{array}{l}\text { Referred } \\
\text { sensations }\end{array}$ & $\begin{array}{l}\text { Phantom } \\
\text { pain }\end{array}$ \\
\hline 1 & $\mathrm{M}$ & 75 & 65 & $\mathrm{R}$ & Yes & 17 & Yes & Yes & Thumb and palm & No \\
\hline 2 & $M$ & 42 & 15 & $L$ & Yes & 9 & Yes & Yes & Full phantom hand & Yes \\
\hline 3 & $M$ & 53 & 36 & $L$ & No & 24 & Yes & Yes & Three fingers & No \\
\hline 4 & $M$ & 65 & 47 & $L$ & No & 22 & Yes & Yes & Palm & No \\
\hline 5 & $M$ & 49 & 28 & $L$ & No & 19 & Yes & Yes & Five fingers & No \\
\hline 6 & $\mathrm{~F}$ & 38 & 5 & $\mathrm{R}$ & Yes & 15 & Yes & Yes & Five fingers & Yes \\
\hline 7 & $M$ & 23 & 1 & $\mathrm{R}$ & Yes & 21 & Yes & Yes & Four fingers & Yes \\
\hline 8 & $M$ & 55 & 34 & $\mathrm{R}$ & Yes & 29 & Yes & Yes & Five fingers & Yes \\
\hline
\end{tabular}

Details of all patients participating in the study.
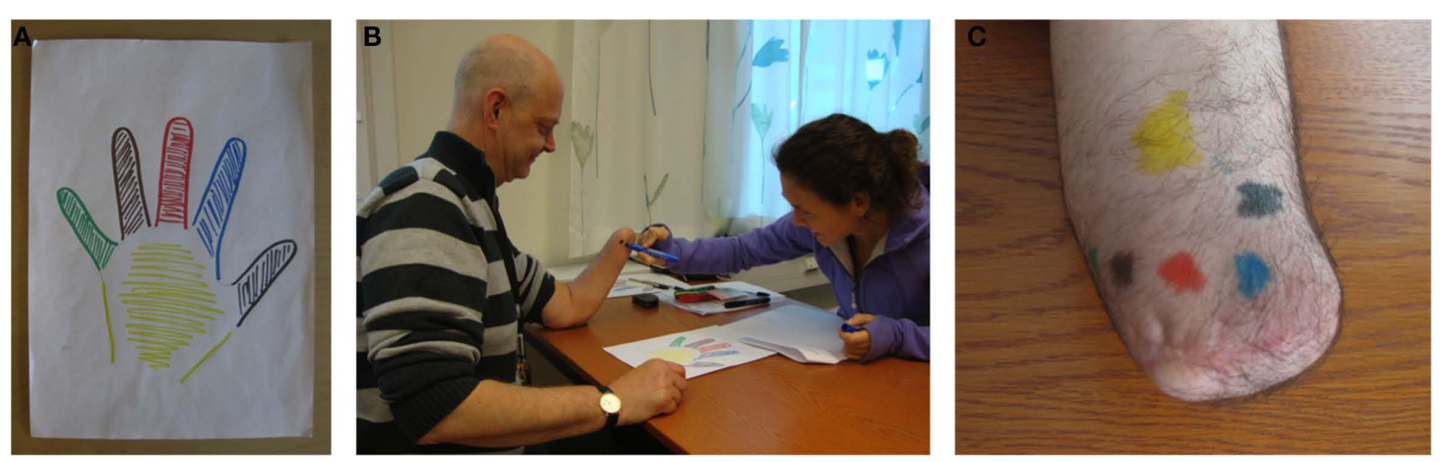

FIGURE 1 | Stump mapping. (A) Drawn hand template used for the stump mapping. (B) For each participant the spots triggering referred sensations in specific parts of the phantom hand were marked on the stump. (C) Example of a stump map. 


\section{EXPERIMENTAL SETUP AND BASIC EXPERIMENTAL PROCEDURES}

All experiments involved a "full-body illusion," and the setup was based on that previously described by Petkova and Ehrsson (2008) as well as Schmalzl and Ehrsson (2011).

\section{Head-mounted displays and CCTV cameras}

During the conduction of the experiments, participants wore a set of (HMDs; Cybermind Visette Pro PAL, Cybermind Interactive, Maastricht, the Netherlands) with a field of view of $71.5^{\circ}$ and a display resolution of $640 \times 480$. The HMDs were connected to two synchronized color CCTV cameras (Panasonic WV-CP484E), which were attached to a helmet worn by a mannequin. The cameras were positioned so as to record the mannequin's body from above (Figure 2), hence providing the participants with a first person perspective view of the mannequin's body which is a crucial factor for the induction of the illusion (Petkova et al., 2011a). The position of the cameras corresponded to that of the mannequin's eyes, and in order to provide the participants with a stereoscopic image the recordings from the left and right video cameras were projected onto the left and right side of the HMDs respectively. In addition, the CCTV camera signals were directly relayed into the HMDs without any external conversion devices, allowing the participants to view the recordings with no noticeable delay.

\section{Mannequin}

For all experiments we used a male mannequin dressed with blue jeans and a white t-shirt. In Experiment 1 the mannequin was intact, whereas in Experiments 2 and 3 the mannequin was missing either its left or right hand, depending on the side of the amputation of each participant, so that the respective arm resembled the stump of an upper limb amputee (Figure 2). Throughout all experiments we used the same mannequin that we used in our previous study on healthy individuals (Schmalzl and Ehrsson, 2011), and therefore the stump length of the mannequin was the same for all participants.

\section{Visuo-tactile stimulation through stroking}

During all experiments participants assumed a standing position and wore the HMDs connected to CCTV cameras as described above. They were instructed to tilt their head forward as if they were looking down at their own body, and therefore viewed the mannequin's body from a first person perspective. Participants were then subjected to visuo-tactile stimulation through stroking. That is, the experimenter contemporarily stroked the mannequin's body (which was in full view) and the participants' body (which was out of view; Figure 2). Hence, the participants visually perceived the touches applied to the mannequin, while experiencing tactile stimulations on their own bodies. Stroking of the mannequin and the participants was performed either in temporal synchrony (Experiment 1 - Condition 1; Experiment 2 - Conditions 1 and 2; Experiment 3 - Conditions 1 and 2) or asynchrony (Experiment 1 - Condition 2). Details about the characteristics, spatial location and duration and of the stroking in each of the experimental conditions are provided below.

\section{EXPERIMENT 1}

In Experiment 1 we used an intact mannequin, and the general experimental procedures were based on those described in Petkova and Ehrsson (2008).

\section{Condition 1 - Synchronous stroking}

In Condition 1, the experimenter stroked the mannequin and the participants synchronously. That is, participants visually perceived the strokes applied to the mannequin at the exact same time and location as they perceived the tactile stimulations on
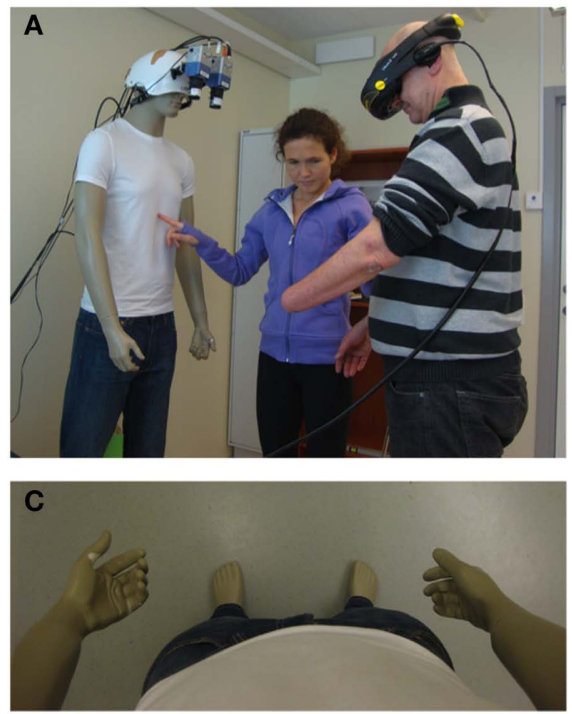

FIGURE 2 | Full-body illusion setup. For the induction of the full-body illusion synchronous strokes were applied to the participants and the mannequin, while the participants were viewing the mannequin's body from a first person perspective through a set of HMDs. (A) Intact
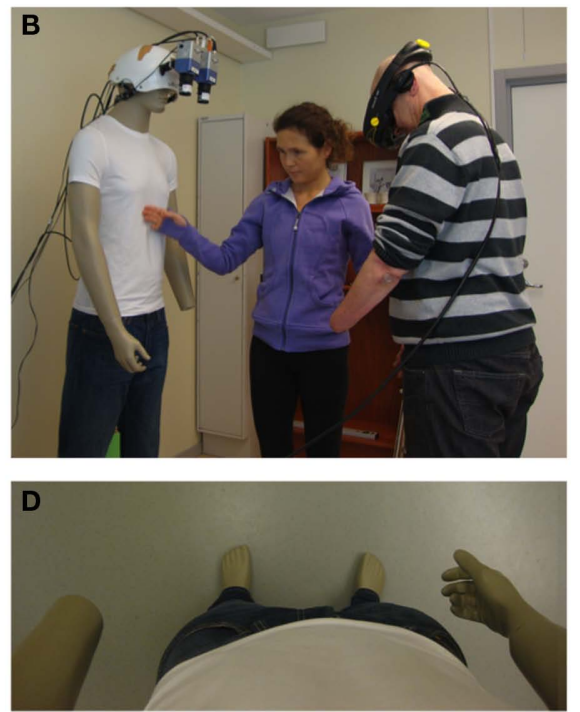

mannequin used in Experiment 1. (B) Amputated mannequin used in Experiments 2 and 3. (C) Intact mannequin seen from the participants' perspective. (D) Amputated mannequin seen from the participants' perspective. 
their own bodies. Such synchronous visuo-tactile stimulation has previously been shown to evoke a "full-body illusion", namely the experience of perceiving the mannequin's body as one's own (Van der Hoort et al., 2001; Petkova and Ehrsson, 2008; Petkova et al., 2011a; Schmalzl and Ehrsson, 2011). Strokes were applied with a frequency of approximately one stroke per second, and the stroke length varied from about $1-5 \mathrm{~cm}$. The temporal stroking pattern was kept irregular in order to avoid expectations about the timing of the visuo-tactile stimulation events in the participants. In regard to the spatial location of the stroking the sequence was as follows: (a) stroking of the torso (45s); (b) stroking of the intact hand $(45 \mathrm{~s})$; (c) stroking of the stump of the participant and the corresponding intact hand of the mannequin ( $45 \mathrm{~s})$. Crucially, the location of strokes applied to the stump was based on the individual stump maps described above. That is, for each participant we chose the spot on the stump that evoked the strongest referred sensations in the phantom hand (for one participant it was the palm of the hand, whereas for all other participants it was a specific point on one of the fingers). That spot was then touched at the same time as the corresponding part of the intact hand of the mannequin. Hence, while all other experimental procedures were kept consistent across participants, the exact spatial location of the stroking according to referred sensations had to necessarily be tailored individually.

\section{Condition 2 - Asynchronous stroking}

In Condition 2, the experimenter stroked the mannequin and the participants asynchronously. That is, participants visually perceived the strokes applied to the mannequin either before or after they perceived the tactile stimulations on their own bodies. The temporal discrepancy between seen and felt touch was about $1 \mathrm{~s}$. All other aspects of the stroking such as frequency, length and spatial location were kept identical to Condition 1. Temporal asynchrony has previously been shown to abolish or at least significantly reduce the feeling of ownership of the mannequin's body (Petkova and Ehrsson, 2008). It therefore represents a valid control condition, allowing one to determine whether potential illusory ownership sensations observed with synchronous stroking are in fact driven by visuo-tactile integration, and not merely by the fact of viewing an artificial body from a first person perspective, or general psychological factors such as suggestibility and task compliance.

Participants experienced each of the experimental conditions once in counterbalanced order. In between each condition the HMDs were removed, and participants were asked to fill out a questionnaire in regard to their experience during the stroking (see below).

\section{Questionnaire}

Following both Condition 1 and 2, each consisting of a total of $135 \mathrm{~s}$ of stroking of the torso, the intact arm and then the stump, participants were asked to complete a questionnaire aimed at capturing the subjective experience of the experimental effects. The questionnaire consisted of 10 statements describing possible perceptual effects that might be elicited by the stroking, and participants were asked to affirm or deny experiencing each effect on a seven-point Likert scale. Six statements were aimed at capturing the illusion of ownership of (a) the mannequin's body as a whole; (b) the mannequin's hand on the side corresponding to the participants' intact hand; (c) the mannequin's hand on the side corresponding to the participants' phantom hand. The remaining four statements were control statements aimed at capturing the participants' suggestibility and task compliance (Figure 3). The order of the questions was randomized across participants in order to avoid order effects. The crucial questions of interest were whether synchronous stroking in Condition 1 would cause amputees to (a) experience ownership of an intact body, and even more importantly (b) experience ownership of the mannequin's hand corresponding to their phantom hand.

\section{Pain rating scale}

Following both Condition 1 and 2, participants who experienced phantom pain on the day of testing were again asked to rate the intensity of their pain on a visual analog scale from 0 to 10 . The aim was to document whether any of the experimental procedures had any effect on phantom pain sensations.

\section{EXPERIMENT 2}

In Experiment 2 the mannequin was missing either its left or right hand (depending on the side of amputation of each participant), so as to resemble and upper limb amputee, and the experimental procedures were based on those described in Schmalzl and Ehrsson (2011).

\section{Induction of the full-body illusion}

Both experimental conditions of Experiment 2 involved the induction of a full-body illusion, which was performed following the exact same procedure as in Experiment 1. That is, participants were initially subjected to synchronous visuo-tactile stimulation of (a) the torso (45s) and (b) the intact hand (45s). With regard to the stroking of the stump however, the two experimental conditions differed from each other.

\section{Condition 1 - Synchronous stroking of the participants' stump and the empty space below the mannequin's stump}

In Condition 1, the experimenter synchronously stroked the participants' stump and empty space below the mannequin's stump (Figure 4). Again, particular emphasis was given to the point eliciting the strongest referred sensations in the phantom hand.

\section{Condition 2 - Synchronous stroking of the participants' stump and the mannequin's stump}

In Condition 2, the experimenter synchronously stroked the participants' stump and the mannequin's stump at corresponding locations. Strokes were spatially distributed so as to cover most of the surface of the stump (Figure 4). However, as in Experiment 1 for each participant particular emphasis was given to the point eliciting the strongest referred sensations in the phantom hand.

Participants experienced each of the experimental conditions once in counterbalanced order. After each condition the HMDs were removed, and participants were asked to fill out a questionnaire in regard to their experience during the stroking (see below). 


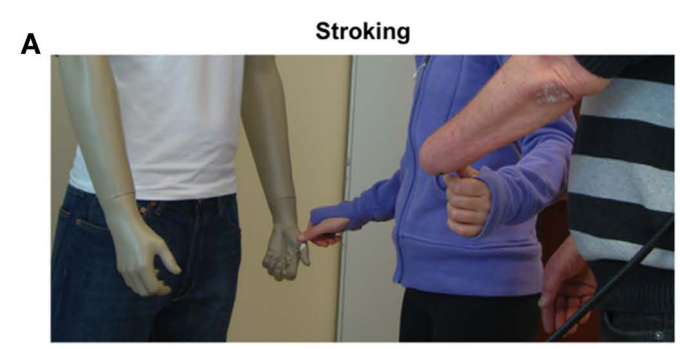

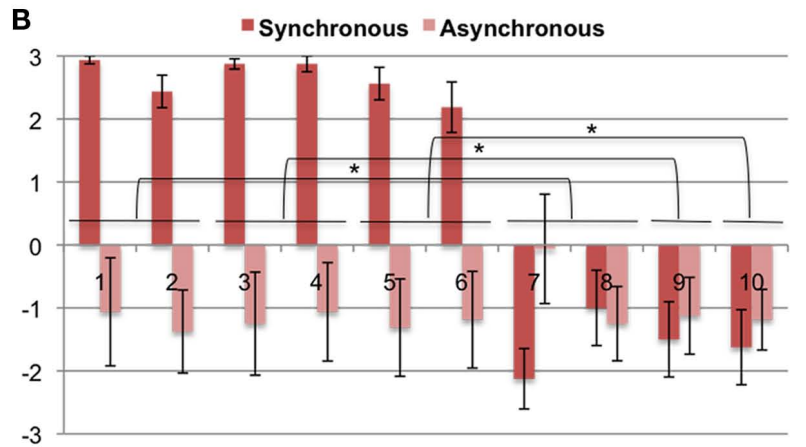

FIGURE 3 | Experiment 1 - Visuo-tactile stimulation followed by questionnaire. (A) Stroking of the trigger points of the participant's stump and the corresponding parts of the mannequin's hand - strokes were applied either in temporal synchrony or asynchrony. (B) The questionnaire consisted of 10 statements: Ownership of the mannequin's body as a whole (Illusion statements 1-2, Control statements 7-8); Ownership of the mannequin's hand corresponding to the participants' intact hand (Illusion statements 3-4, Control statement 9); Ownership of the mannequin's hand
1. It seemed as if I could feel the touch given to the mannequin's body

2. It felt like the mannequin's body was my own body

3. It seemed as if I could feel the touch given to the mannequin's right hand (or hand corresponding to intact hand)

4. It felt like the mannequin's right hand was my own right hand (or hand corresponding to intact hand)

5. It seemed as if I could feel the touch given to the mannequin's left hand (or hand corresponding to amputated hand)

6. It felt like the mannequin's left hand was my own left hand (or hand corresponding to amputated hand)

7. It felt as if I had two bodies at the same time

8. The mannequin's body started to change appearance (visually) and look the way my own real body looks

9. My intact hand started to feel as if it was made out of rubber

10. My stump started to feel as if it was made out of rubber corresponding to the participants' amputated hand (Illusion statements 5-6, Control statement 10). Participants were asked to affirm or deny each statement on a seven-point Likert scale $(+3=$ Strongly agree; $-3=$ Strongly disagree). The graph shows the mean group values with SD bars. Wilcoxon Signed Rank Tests were performed between illusion statements and control statements, as well as between the Synchronous and Asynchronous conditions - for more details see the Sections "Materials and Methods" and "Results." * $p<0.05$.

\section{Questionnaire}

As in Experiment 1, following both Condition 1 and 2 participants were asked to complete a questionnaire aimed at capturing the subjective experience of the experimental effects. The questionnaire consisted of six statements, and participants were again asked to affirm or deny experiencing each effect on a seven-point Likert scale. Two statements were intended to capture the overall illusory experience of perceiving the mannequin's body as one's own, two statements directly referred to the perceived location of the phantom hand, and the remaining two statements were control statements aimed at capturing the participants' suggestibility and task compliance (Figure 4). The order of the questions was randomized across participants in order to avoid order effects. The crucial questions of interest were whether participants would report feeling their phantom hand to be in a different location with respect to the stump of the mannequin in Condition 1 vs. Condition 2. Given that the experienced phantom sensations differed for each of the participants, and that the tactile stimulation was targeted so as to evoke referred sensations in a specific point of the phantom hand, the statements referring to the perceived position of the phantom hand were adapted accordingly for each participant. That is, the general term phantom hand was replaced with the specific part or finger of the phantom hand that was targeted for each participant (e.g., phantom thumb).

\section{Pain rating scale}

Following both Condition 1 and 2, participants who experienced phantom pain on the day of testing were again asked to rate the intensity of their pain on a visual analog scale from 0 to 10 .

\section{EXPERIMENT 3}

In Experiment 3 the setup as well as stroking procedures were exactly the same as in Experiment 2 for both Condition 1 and Condition 2. This time however, participants experienced each of the experimental conditions three times in counterbalanced order, and at the end of each stroking session they were asked to perform a visual judgment task to indicate the perceived position of their phantom hand (see below). The task was adapted from Schmalzl and Ehrsson (2011).

\section{Visual judgment task}

After each stroking session, consisting of a total of $135 \mathrm{~s}$ stroking of the torso, the intact arm and then the stump (Condition 1 or 2), participants were asked to perform a visual judgment task in which they had to visually judge the perceived location of their phantom hand. The purpose of this task was to obtain an objective measure of the experimental effect found in Experiment 2, namely a subjectively experienced difference in the perceived position of the phantom hand in Condition 1 (below the stump) vs. Condition 2 (inside the stump; see Results section). The task was performed as 


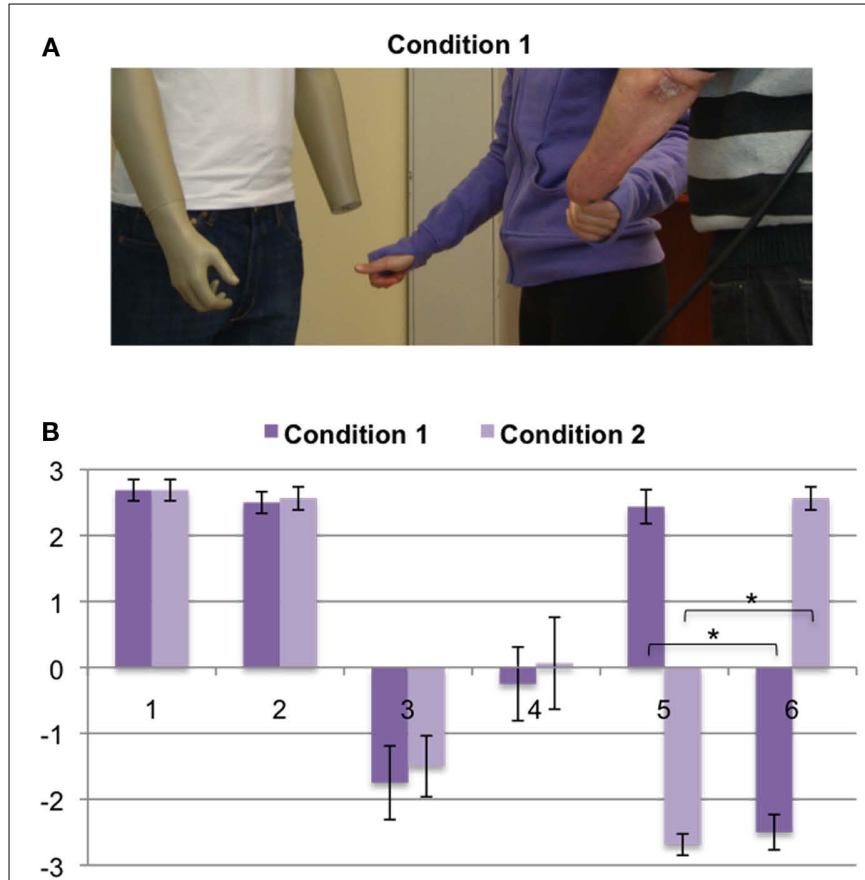

FIGURE 4 | Experiment 2 - Visuo-tactile stimulation followed by questionnaire. (A) Stroking conditions: Condition 1 - simultaneous stroking of the trigger points of the participant's stump and the empty space below the mannequin's stump; Condition 2 - simultaneous stroking of the trigger points of the participant's stump the mannequin's stump. (B) The questionnaire consisted of six statements: Experience of the full-body illusion (Illusion statements 1-2, Control statements 3-4); Perceived location of the phantom hand (Condition 1: Illusion statement
1. It seemed as if I could feel the touch given to the mannequin

2. It felt like the mannequin's body was my own body

3. It felt as if I had two bodies at the same time

4. The mannequin's body started to change appearance (visually) and look the way my own real body looks

5. It felt as if my phantom hand was located below the stump of the mannequin

6. It felt as if my phantom hand was located inside the stump of the mannequin follows: after each stroking session participants were asked to look at "their" stump, that is the stump of the mannequin, through the HMDs. The experimenter then moved a ruler from the floor up toward the stump, and participants verbally instructed the experimenter to adjust the height of the ruler so it would match the height at which they felt their phantom hand to be. The absolute height of that location was then manually recorded by the experimenter on a measuring band attached to a stand (measurements were rounded up to 0.5 of a centimeter). A spirit level ruler was used in order to assure its horizontal alignment when recording the height on the measurement band (Figure 5). In between each stroking session participants were given a short break during which the cameras were occluded, and then the procedure was repeated. The crucial question of interest was whether there would be a significant difference in the visually judged height of the perceived position of the phantom hand following the two stroking conditions, reflecting the subjectively reported difference observed in Experiment 2. Again, as in Experiment 2, the specific instructions were adapted for each participant according to the specific part of the phantom hand that was targeted during the stroking (e.g., judgment of the height of the phantom thumb).

\section{DATA ANALYSIS}

All acquired data was tested for normal distribution using the Shapiro and Wilk Test. The questionnaire data of Experiments 1 and 2 did not pass the test for normality, and was therefore analyzed using non-parametric statistics. In contrast, the data obtained from the visual judgment task did pass the test for normality, allowing for the use of parametric statistics. All analyses were based on a priory hypotheses, and hence consisted of planned comparisons. For questionnaire data, comparisons were performed between the illusion questions and their respective control questions, or between the two stroking conditions of each experiment (Conditions 1 and 2 described above), using Wilcoxon Signed Rank Tests. For the visual judgment task, comparisons were performed between Condition 1 and Condition 2 using a Paired Samples $t$-test. Since all analyses were based on a priory hypotheses, no post hoc corrections for multiple comparisons were performed. For all analyses, alpha was set to 0.05 .

\section{RESULTS}

Below we will report the results of each of the experiments, followed by a summary of the participants' ratings on the phantom pain rating scale, which was administered after Experiments 1 and 2.

\section{EXPERIMENT 1}

Aim

The aim of Experiment 1 was to investigate whether upper limb amputees can experience a full-body illusion with an intact mannequin, and specifically address the question of whether they 
A

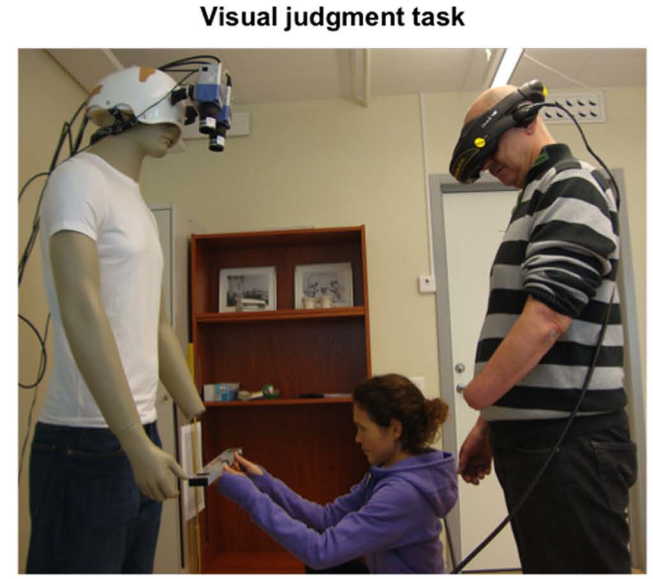

B

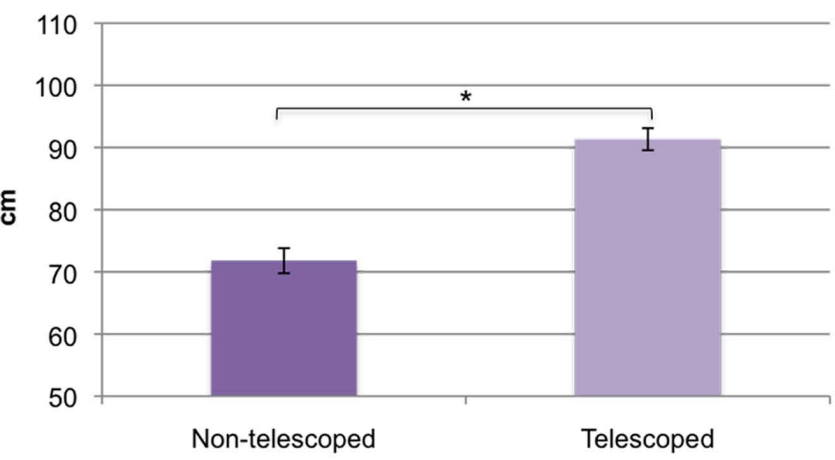

Condition
C

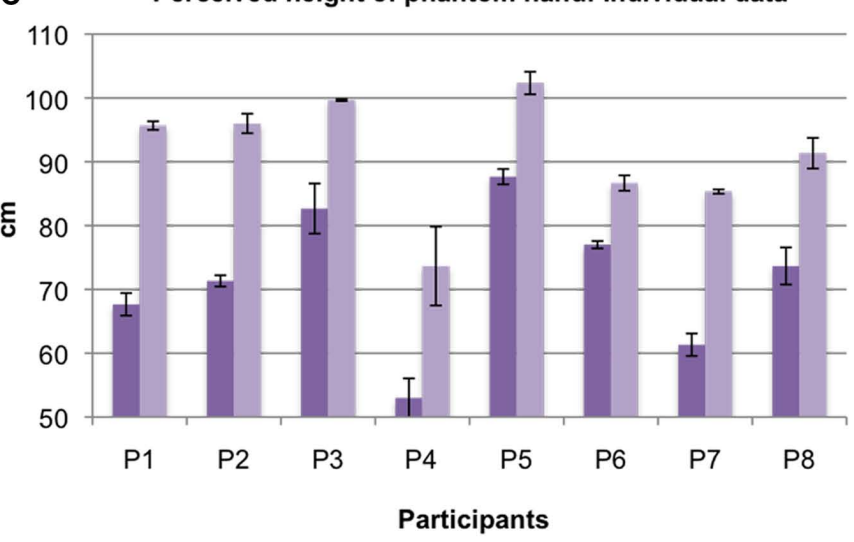

phantom hand to be. (B) Mean height of the perceived position of the phantom hand in the Non-telescoped and Telescoped condition (Paired Samples $t$-test $-p<0.001)$. (C) Individual data.
FIGURE 5 | Experiment 3 - Visual judgment task. (A) At the end of each stroking session participants verbally instructed the experimenter to adjust the height of a ruler so it would match the height at which they felt their
Illusion statements mean score vs. control statements mean score-Wilcoxon Signed Rank Test: synchronous: $Z=-2.536$, $p=0.011$; Asynchronous: $Z=-0.957, p=0.339)$.

(b) Ownership of the mannequin's hand corresponding to the participants' intact hand (Illusion statements 3-4, Control statement 9)

Participants affirmed experiencing ownership of the mannequin's hand corresponding to their intact hand in the Synchronous condition, but not in the Asynchronous condition (Illusion statements mean score vs. control statement-Wilcoxon Signed Rank Test: synchronous: $Z=-2.527$, $p=0.012$; Asynchronous: $Z=-0.213, p=0.832$ ).

(c) Ownership of the mannequin's hand corresponding to the participants' amputated hand (Illusion statements 5-6, Control statement 10)

Participants affirmed experiencing ownership of the mannequin's hand corresponding to their amputated hand in the Synchronous condition, but not in the Asynchronous condition (Illusion statements mean score vs. control statement Wilcoxon Signed Rank Test: synchronous: $Z=-2.536, p=$ 0.011; Asynchronous: $Z=-0.271, p=0.786)$. Importantly, 
the ownership ratings for the hand corresponding to the amputated hand were not significantly different from those for the hand corresponding to the intact hand (Illusion statement for intact hand vs. illusion statement for amputated hand-Wilcoxon Signed Rank Test: synchronous: $Z=-1.753$, $p=0.080$ ).

\section{EXPERIMENT 2}

\section{Aim}

The aim of Experiment 2 was to investigate whether differential visuo-tactile stimulation within the context of the full-body illusion can be used to manipulate the perceived position of phantom hands. Specifically, we aimed to investigate whether it is possible to temporarily revoke telescoping sensations, and have amputees experience their phantom hand to be located outside the stump, without involving any visualization of an artificial hand.

\section{Questionnaire results}

In Experiment 2, the statements of the questionnaire specifically referred the following aspects: (a) experience of the full-body illusion; (b) perceived location of the phantom hand. As in Experiment 1, the comparison between illusion and control statements, as well as the comparisons between stroking conditions for each of these aspects was performed independently (Figure 4).

(a) Experience of the full-body illusion (Illusion statements 1-2, Control statements 3-4)

Following both Condition 1 (stimulation on the stump) and Condition 2 (stimulation below the stump), participants clearly affirmed experiencing the full-body illusion. (Illusion statements mean score vs. control statements mean score-Wilcoxon Signed Rank Test: Condition 1: $Z=-2.533$, $p=0.011$; Condition $2: Z=-2.536, p=0.011)$. There was no difference in the perceived strength of the full-body illusion in the two conditions (Condition 1 vs. Condition $2-$ Wilcoxon Signed Rank Test: illusion statements mean score: $Z=-1.000$, $p=0.317$; Control statements mean score: $Z=-0.742$, $p=0.458)$.

(b) Perceived location of the phantom hand (For Condition 1: Illusion statement 5, Control statement 6; For Condition 2: Illusion statement 6 , Control statement 5)

There was a significant difference in the perceived position of the phantom hand in the two stroking conditions. In Condition 1, participants clearly affirmed that they perceived their phantom hand to be located below the stump (Illusion statement vs. control statement - Wilcoxon Signed Rank Test: $Z=-2.565, p=0.010)$. In Condition 2, participants clearly affirmed that they perceived their phantom hand to be located inside the stump (Illusion statement vs. control statement - Wilcoxon Signed Rank Test: $Z=-2.558, p=0.011$ ). When comparing the participants' responses between Conditions 1 and 2, there was a significant difference for both the statement referring to the phantom hand being perceived below the stump (Wilcoxon Signed Rank Test: $Z=-2.565$, $p=0.010$ ), and the statement referring to the phantom hand being perceived inside the stump (Wilcoxon Signed Rank Test: $Z=-2.555, p=0.011$ ).

\section{EXPERIMENT 3}

Aim

The aim of Experiment 3 was to obtain an objective measure of the experimental effect found in Experiment 2, namely the difference in the perceived position of the phantom hand in Condition 1 (below the stump) vs. Condition 2 (inside the stump). Specifically, we aimed to investigate whether there would be a significant difference in the visually judged height of the perceived position of the phantom hand following the differential stroking conditions. Given the results of the subjective reports in Experiment 2, hereafter Condition 1 will be referred to as "Non-telescoped" condition, and Condition 2 will be referred to as "Telescoped" condition.

\section{Visual judgment task results}

The results of the visual judgment task showed a significant difference in the visually judged height of the perceived position of phantom hand in Non-telescoped condition vs. Telescoped condition (Paired Samples $t$-test: $t=9.244, p=0.001$; Figure 5).

\section{Pain rating scale results}

Four of the participants ( $P 2,6,7$, and 8$)$ reported to experience phantom pain at the time of testing, and were therefore asked to rate the intensity of their pain after each experimental condition of Experiments 1 and 2. The effect of the experimental procedures on phantom pain sensations varied across participants. Participant 2 reported a very strong attenuation effect of the Synchronous condition of Experiment 1 and the Non-telescoped condition of Experiment 2, during both of which he reported that his phantom pain had completely disappeared (rating the pain as zero). Participant 6 reported a slight reduction of pain during both the Synchronous and Asynchronous conditions of Experiment 1, and a substantial reduction of pain during the Non-telescoped condition of Experiment 2. Participants 7 and 8 on the other hand did not report any reduction in phantom pain during any of the experimental conditions. A summary of the ratings is provided in Figure 6, and a further discussion of the findings is provided in the Discussion section below.

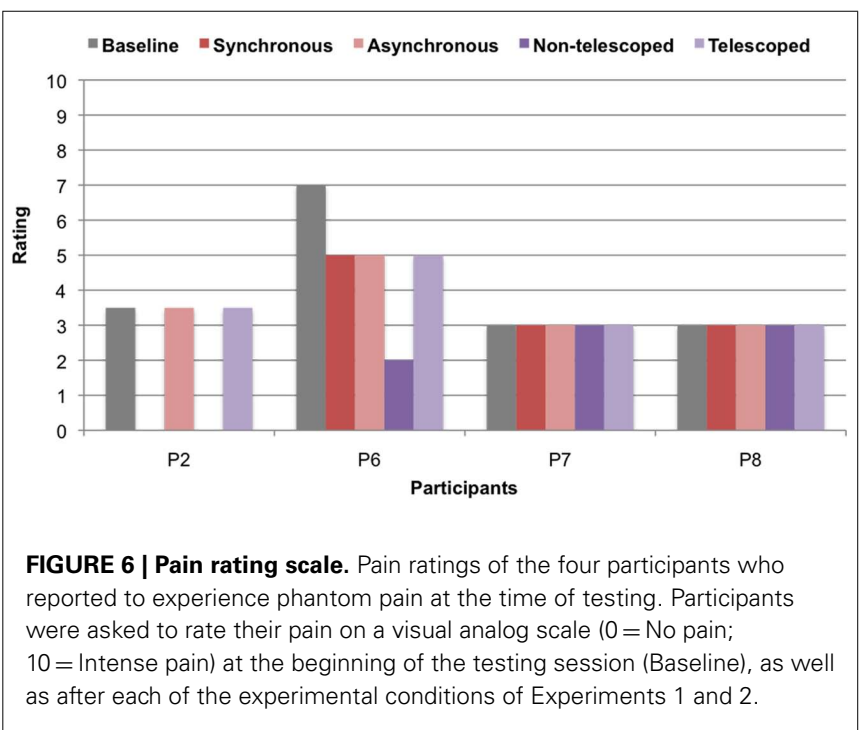




\section{DISCUSSION}

In the current study we set out to investigate whether the fullbody illusion can be used to induce ownership of an intact body in upper limb amputees, whether it is possible to manipulate the perceived position of phantom hands without involving any visualization of an intact hand (as in the rubber hand illusion or MVF therapy), and whether any of these experimental procedures can have an effect on phantom pain. We will now discuss our results in relation to each of these questions.

\section{CAN AMPUTEES EXPERIENCE OWNERSHIP OF AN INTACT BODY, AND REFERRAL OF TOUCH FROM AN ARTIFICIAL HAND, IN THE CONTEXT OF THE FULL-BODY ILLUSION?}

According to the questionnaire data of Experiment 1, following synchronous visuo-tactile stimulation amputees affirmed experiencing ownership of an intact artificial body, as well as referral of touch from both hands of the mannequin. The fact that amputees can experience ownership of an intact body is actually not that unexpected. Amputees have had the experience of living with an intact body prior to their amputation, and through the use of prosthetic limbs many of them continue to experience an at least visually intact body on a daily basis following the amputation as well. Also, the demonstration of the fact that amputees can experience referral of touch from an artificial hand is not a novel finding in itself. In fact, it has been previously demonstrated with both a rubber hand (Ehrsson et al., 2008) and a robotic hand (Marasco et al., 2011 - targeted reinnervation amputees; Rose'n et al., 2009normal upper limb amputees). So why did we choose to initially conduct the full-body illusion with an intact mannequin in Experiment 1? Firstly, we wanted to establish that participants would respond to synchronized visuo-tactile stimulation and experience the full-body illusion as such, as both Experiments 2 and 3 were to be based on this procedure. Secondly, the choice of using an intact mannequin was based on the fact that we wanted to determine whether we could replicate the findings of Ehrsson et al. (2008), and evoke referred touch based on individual stump maps, also in the context of the full-body illusion. Establishing this fact was also a prerequisite for the more sophisticated experimental manipulations in Experiment 2 and 3. In regard to the second point, Experiment 1 yielded very interesting results. Not only were we able to replicate the induction of ownership sensations for the artificial hand, but the average ratings for ownership as well as referral of touch from the artificial hand were actually much higher in our study compared to those obtained with the rubber hand illusion by Ehrsson et al. (2008). Of course, comparisons between studies with different patient groups are to be done with caution, but we thought it would nevertheless be of interest to discuss possible reasons for the differential findings. One possible reason is related to the employed experimental procedures. Namely, it can be hypothesized that the strength of the overall bodily coherence experienced in the context of the fullbody illusion (Petkova and Ehrsson, 2008) facilitates the induction of ownership sensations of the artificial hand compared to the rubber hand illusion. We know that during the full-body illusion multisensory signals causing ownership sensations in regard to a particular body part facilitate ownership sensations of other (non-stimulated) body parts (Petkova and Ehrsson, 2008; Petkova et al., 2011b), and these facilitating effects across body parts could be at play also in our setup (e.g., from the torso to the hand). In addition, the induction might be facilitated by the fact that in the full-body illusion the artificial hand is in direct continuation with the arm, whereas in the rubber hand illusion the hand is displaced $15-20 \mathrm{~cm}$ medial from the hidden real hand creating an additional visuo-proprioceptive spatial mismatch. Another possible reason is related to the two patient groups. Ehrsson et al.'s (2008) study also included patients who did not have specific stump maps, and hence no trigger points that evoke specific referred sensations. For these patients, strokes were simply applied to the middle of the stump as opposed to precisely targeted locations with respect to the artificial hand, which can be expected to be less effective. As a matter of fact, in the discussion of their paper Ehrsson et al. (2008) report that the patients without stump maps had lower ownership ratings than the ones with stump maps. Although this difference was not significant, it surely lowered the average score of the group. Lastly, the full-body illusion with an intact mannequin allows one to compare the strength of ownership sensations for the artificial hand corresponding to the missing hand (triggered by touching the points on the stump map) and the intact hand (triggered by touching the actual hand) within the same stroking session. In our group of patients, there was no significant difference between the ownership ratings for the two hands. Again, this contrasts the findings of Ehrsson et al. (2008) who performed the rubber hand illusion for each hand individually in separate stroking sessions, and found the ratings for the amputated hand to be significantly lower than those for the intact hand. This is of particular interest and confirms the effectiveness of precisely targeted tactile stimulation on the stump in the context of the full-body illusion and the therewith evoked overall bodily coherence.

\section{CAN THE FULL-BODY ILLUSION WITH AN AMPUTATED MANNEQUIN BE USED TO MANIPULATE THE PERCEIVED POSITION OF PHANTOM HANDS, AND TEMPORARILY REVOKE TELESCOPING SENSATIONS WITHOUT INVOLVING THE VISUALIZATION OF AN INTACT HAND?}

The questionnaire data of Experiment 2 showed that, depending on the stroking condition, participants affirmed experiencing their phantom hand to be located either below the stump, or inside the stump and therefore remain telescoped. These subjective ratings were then confirmed in Experiment 3, in which participants judged the perceived height of their phantom hand in a visual judgment task. The effect was very robust, with all participants showing the proprioceptive drift in the Non-telescoped condition. To our knowledge, this is the first demonstration of a systematic and "dynamic" manipulation of the perceived position of phantom limbs within the same experimental session. Moreover, our findings show that the temporary revoking of telescoping sensations does not necessarily have to involve the visualization of an intact hand (as in the rubber hand illusion or MVF therapy), but that it can also be obtained through mere referral of touch from the spatial location corresponding to that previously occupied by the intact hand (i.e., the "pulled out" phantom below the stump). How can this be explained? In the case of experimental manipulations involving the visualization of an intact hand, such as the rubber hand illusion in Ehrsson et al.'s (2008) study and 
Experiment 1 of our current study, illusory ownership of an artificial hand in amputees is likely to be caused by similar cognitive and neural mechanisms as those underlying the classical rubber hand illusion in healthy individuals (Botvinick and Cohen, 1998; Botvinick, 2004; Ehrsson et al., 2004, 2005a). That is, if stroking is performed according to a specific stump map, the brain would receive congruent multisensory information as the participants feel touch on their phantom hand at the exact same time and in the exact same location as they see touch on the artificial hand. The integration of this information by multisensory brain areas would then lead to illusory ownership sensations of the artificial hand. As pointed out by Ehrsson et al. (2008) though, given the cortical re-organization occurring in amputees (Ramachandran and Hirstein, 1998; Flor et al., 2006), the thalamo-cortical and corticocortical pathways by which the tactile information from the stump reaches the deafferented primary sensorimotor cortex and consequently multisensory areas in premotor and intraparietal cortices, would be reorganized with respect to those of healthy individuals. In any case, there are two important additional factors that are relevant for the discussion about the underlying mechanisms of the "pulling-the-phantom-out-of-the-stump" effect. Firstly, the touch stimulation is applied to the empty space below the stump rather than on a visible hand. Secondly, the representation of the (phantom) hand is stretched out and elongated so that the phantom is now experienced to be of normal length, i.e., no longer telescoped. We will now discuss these two points in turn. From rubber hand illusion experiments with healthy participants we know that illusory ownership sensations are disrupted if the artificial hand is replaced by an object not resembling a human hand (Tsakiris et al., 2010). From our own recent work involving the full-body illusion however, we also know that referral of touch can still be evoked if the artificial hand is missing completely (Schmalzl and Ehrsson, 2011). That is, if the hand of the mannequin is "replaced" by empty space, participants experience touch from an invisible hand located at the point in space in which they visually see the stimulation occur. So, as opposed to the classical rubber hand or full-body illusion, what we are dealing with here is the induction of referral of touch from a certain location in space without the induction of ownership of a visible artificial hand. And according to the findings of our previous study and the current study, this seems to work for both real hands of healthy individuals and phantom hands of amputees. With respect to this latter point, we can conclude that the representation of the phantom arm seems to be "stretched" out, with the consequence of the phantom arm being perceived as longer. We know from several studies that congruent multisensory signals can lead to illusory sensations of elongated limbs or other body parts in the healthy brain (Craske et al., 1984; Lackner, 1988). Although little is known about the central representation of the relative length of limbs or body segments, it can be assumed that this information is derived from cortical multisensory signals originating from several body segments, as there are no peripheral receptors in the body that signals the size (or length) of body segments (Gandevia and Phegan, 1999). One imaging study suggests that the posterior parietal cortex has an important role in this estimation of the size of individual body parts (Ehrsson et al., 2005b), which is consistent with the neurological observation that migraine or focal epilepsy originating from the parietal lobe can produce the "Alice in Wonderland syndrome" which causes people to experience body parts to be expanding or shrinking in size (Lippman, 1952; Sveinbjornsdottir and Duncan, 1993; Todd, 1955). In addition, a study using neuromagnetic source imaging (Schaefer et al., 2007) also showed that the illusory sensation of an elongated arm was associated with a change in the cortical distance between the representation of individual fingers in primary somatosensory cortex (S1), suggesting the involvement of this area in the generation and maintenance of the illusion. Which of these neural mechanisms are at play in the manipulation of the perceived position of phantom limbs in our study remains speculative. In any case however, our behavioral results show that dynamic re-calibration of hand position and arm length by dynamic multisensory input in amputees is sufficient to override the neuro-anatomical mechanisms that led to the telescoping in the first place. This is interesting as it suggest that phantom limbs are susceptible to the same multisensory illusions of perceived limb elongation as intact arms, emphasizing the similarities between the central representations of phantom limbs and intact limbs (in line with the ideas expressed by Ramachandran and Hirstein, 1998).

\section{DO ANY OF THESE EXPERIMENTAL PROCEDURES HAVE AN EFFECT ON PHANTOM PAIN SENSATIONS?}

According to the literature, telescoping tends to be related to increased levels of phantom pain (Flor et al., 2006). Out of the eight participants of our study, four reported to experience phantom pain at the time of testing, and two reported relief from pain following specific experimental procedures. The first patient (P2) reported that his phantom pain completely vanished during the two experimental conditions evoking the sensation of a nontelescoped phantom, namely in the Synchronous Condition of Experiment 1, and the Non-telescoped Condition of Experiment 2. This was remarkable, as he had been experiencing constant phantom pain since his amputation 15 years prior to the current study. Moreover, his phantom pain was resistant to pharmacological treatment, and therefore monitored through an electrical stimulator implant to his spinal cord. The second patient (P6) experienced a substantial reduction of pain in the Non-telescoped condition of Experiment 2. Hence, interestingly for her the reduction of pain was more pronounced without visualization than with visualization of an intact hand. P6 did however also report a slight reduction of pain compared to baseline in all other experimental conditions. On questioning, she reported that these ratings reflected the fact that any tactile stimulation on the stump in itself tends to reduce her phantom pain sensations. In terms of the time course of the effects, the two patients also differed from each other. For P2 the pain returned to baseline levels immediately after the stroking was terminated. P6 in contrast reported that the sensation of relief persisted for a few minutes before the pain gradually came back. Of course, our sample size is too small to draw any definite conclusions in regard to the effects of the current experimental manipulation on phantom pain. However, the responses of the two patients lead to two interesting observations: neither the visualization of an intact hand (as in the rubber hand illusion) nor illusory movement of the phantom (as in the MVF), 
seem to be necessary factors for the temporary relief of phantom pain.

\section{CONCLUSION}

In sum, our current study demonstrates how the full-body illusion can be used to manipulate the perceived position of phantom limbs in amputees, and that telescoping sensations can be temporarily revoked without involving any visualization of an intact hand or illusory movement of the phantom hand. It also provides preliminary evidence for the fact that these manipulations can have an effect on phantom pain sensations. These observations open up interesting avenues for a number of potential future investigations. From a theoretical perspective, it would be of great interest to conduct neuroimaging studies

\section{REFERENCES}

Botvinick, M. (2004). Neuroscience. Probing the neural basis of body ownership. Science 305, 782-783.

Botvinick, M., and Cohen, J. (1998). Rubber hands "feel" touch that eyes see. Nature 391, 756.

Carlen, P. L., Wall, P. D., Nadvorna, H., and Steinbach, T. (1978). Phantom limbs and related phenomena in recent traumatic amputations. $\mathrm{Neu}$ rology 28, 211-217.

Chan, B. L., Witt, R., Charrow, A. P., Magee, A., Howard, R., Pasquina, P. F., Heilman, K. M., and Tsao, J. W. (2007). Mirror therapy for phantom limb pain. N. Engl. J. Med. 357, 2206-2207.

Craske, B., Kenny, F. T., and Keith, D. (1984). Modifying an underlying component of perceived arm length: adaptation of tactile location induced by spatial discordance. $J$. Exp. Psychol. Hum. Percept. Perform. 10, 307-317.

Ehrsson, H. H., Holmes, N. P., and Passingham, R. E. (2005a). Touching a rubber hand: feeling of body ownership is associated with activity in multisensory brain areas. J. Neurosci. 25, 10564-10573.

Ehrsson, H. H., Kito, T., Sadato, N., Passingham, R. E., and Naito, E. (2005b). Neural substrate of body size: illusory feeling of shrinking of the waist. PLoS Biol. 3, e412. doi:10.1371/journal.pbio.0030412

Ehrsson, H. H., Rosén, B., Stockselius, A., Ragnö, C., Köhler, P., and Lundborg, G. (2008). Upper limb amputees can be induced to experience a rubber hand as their own. Brain 131, 3443-3452.

Ehrsson, H. H., Spence, C., and Passingham, R. E. (2004). That's my hand! Activity in premotor cortex reflects feeling of ownership of a limb. Science 305, 875-877.

Flor, H., Nikolajsen, L., and Jensen, T. S. (2006). Phantom limb pain: a case of maladaptive CNS plasticity. Nat. Rev. Neurosci. 7, 873-881.

Gandevia, S. C., and Phegan, C. M. L. (1999). Perceptual distortions of the human body image produced by local anaesthesia, pain and cutaneous stimulation. J. Physiol. 514, 609-616.

Giummarra, M. J., Gibsonb, S. J., Georgiou-Karistianisa, N., and Bradshawa, J. L. (2007). Central mechanisms in phantom limb perception: the past, present and future. Brain Res. Rev. 54, 219-232.

Kooijman, C. M., Dijkstra, P. U., Geertzen, J. H. B., Elzinga, A., and van der Schans, C. P. (2000). Phantom pain and phantom sensations in upper limb amputees: an epidemiological study. Pain 87, 33-41.

Lackner, J. R. (1988). Some proprioceptive influences on the perceptual representation of body shape and orientation. Brain 111, 281-297.

Lippman, C. W. (1952). Certain hallucinations peculiar to migraine. J. Nerv. Ment. Dis. 116, 346-335.

Marasco, P. D., Kim, K., Colgate, J. E., Peshkin, M. A., and Kuiken, T. A. (2011). Robotic touch shifts perception of embodiment to a prosthesis in targeted reinnervation amputees. Brain 134, 747-758.

Petkova, V. I., and Ehrsson, H. H. (2008). If I were you: perceptual illusion of body swapping. PLoS ONE 3, e3832. doi:10.1371/journal.pone.0003832

Petkova, V. I., Khoshnevis, M., and Ehrsson, H. H. (2011a). The perspective matters! Multisensory

to elucidate the differential neural mechanisms underlying the illusory ownership of artificial limbs and those underlying the illusory induced change of the perceived position of phantom limbs in amputees. From a clinical perspective, our results set the scene for further studies with larger groups of patients aimed at systematically evaluating the respective effect of illusory movement, visualization and referred touch on phantom pain relief.

\section{ACKNOWLEDGMENTS}

Many thanks to all the patients who participated in this study. This work was funded by the Swedish Foundation for Strategic Research, the European Research Council, and the Human Frontiers of Science Program.

integration in egocentric reference frames determines full body ownership. Front. Psychol. 2:35. doi:10.3389/fpsyg.2011.00035.

Petkova, V. I., Björnsdotter, M., Gentile, G., Jonsson, T., Li, T. Q., and Ehrsson, H. H. (2011b). From part to wholebody ownership in the multisensory brain. Curr. Biol. 21, 1-5.

Ramachandran, V. S., Brang, D., and McGeoch, P. D. (2009). Size reduction using mirror visual feedback (MVF) reduces phantom pain. $\mathrm{Neu}$ rocase $15,357-360$.

Ramachandran, V. S., and Hirstein, W. (1998). The perception of phantom limbs: the D.O. Hebb lecture. Brain 121, 1603-1630.

Ramachandran, V. S., RogersRamachandran, D., and Cobb, S. (1995). Touching the phantom limb. Nature 377, 489-490.

Rose'n, B., Ehrsson, H. H., Antfolk, C. Cipriani, C., Sebelius, F., and Lundborg, G. (2009). Referral of sensation to an advanced humanoid robotic hand prosthesis. Scand. J. Plast. Reconstr. Surg. Hand Surg. 99999, 1-7.

Schaefer, M., Flor, H., Heinze, H. J., and Rottea, M. (2007). Morphing the body: illusory feeling of an elongated arm affects somatosensory homunculus. Neuroimage 36, 700-705.

Schmalzl, L., and Ehrsson, H. H. (2011). Experimental induction of a perceived "telescoped" limb using a full-body illusion. Front. Hum. Neurosci. 5:34. doi:10.3389/fnhum.2011.00034.

Sveinbjornsdottir, S., and Duncan, J. S. (1993). Parietal and occipital lobe epilepsy: a review. Epilepsia 34, 493-521.
Todd, J. (1955). The syndrome of Alice in Wonderland. Can. Med. Assoc. J. 73, 701-704.

Tsakiris, M., Carpenter, L., James, D., and Fotopoulou, A. (2010). Hands only illusion: multisensory integration elicits sense of ownership for body parts but not for noncorporeal objects. Exp. Brain Res. 204, 343-352.

Van der Hoort, B., Guterstam, A., and Ehrsson, H. H. (2001). Being Barbie: the size of one's own body determines the perceived size of the world. PLoS ONE 6, e20195. doi:10.1371/journal.pone. 0020195

Conflict of Interest Statement: The authors declare that the research was conducted in the absence of any commercial or financial relationships that could be construed as a potential conflict of interest.

Received: 05 September 2011; accepted: 10 October 2011; published online: 01 November 2011.

Citation: Schmalzl L, Thomke E, Ragnö $C$, Nilseryd $M$, Stockselius A and Ehrsson HH (2011) "Pulling telescoped phantoms out of the stump": Manipulating the perceived position of phantom limbs using a full-body illusion. Front. Hum. Neurosci. 5:121. doi: 10.3389/fnhum.2011.00121 Copyright (C) 2011 Schmalzl, Thomke, Ragnö, Nilseryd, Stockselius and Ehrsson. This is an open-access article subject to a non-exclusive license between the authors and Frontiers Media SA, which permits use, distribution and reproduction in other forums, provided the original authors and source are credited and other Frontiers conditions are complied with. 